\title{
Analytic Solutions to 3-D Finite Deformation Problems Governed by St Venant-Kirchhoff Material
}

\author{
David Yang Gao ${ }^{1,2}$ \& Eldar Hajilarov ${ }^{1}$ \\ 1. Faculty of Science and Technology,Federation University Australia, Mt Helen, VIC 3353, \\ Australia. \\ 2. Research School of Engineering, Australian National University, Canberra, Australia
}

\begin{abstract}
This paper presents a detailed study on analytical solutions to a general nonlinear boundary-value problem in finite deformation theory. Based on canonical duality theory and the associated pure complementary energy principle in nonlinear elasticity proposed by Gao in 1999, we show that the general nonlinear partial differential equation for deformation is actually equivalent to an algebraic (tensor) equation in stress space. For St Venant-Kirchhoff materials, this coupled cubic algebraic equation can be solved principally to obtain all possible solutions. Our results show that for any given external source field such that the statically admissible first Piola-Kirchhoff stress field has no-zero eigenvalues, the problem has a unique global minimal solution, which is corresponding to a positive-definite second Piola-Kirchhoff stress $\mathbf{S}$, and at most eight local solutions corresponding to negative-definite S. Additionally, the problem could have 15 unstable solutions corresponding to indefinite $\mathbf{S}$. This paper demonstrates that the canonical duality theory and the pure complementary energy principle play fundamental roles in nonconvex analysis and finite deformation theory.
\end{abstract}

Keywords: Nonlinear elasticity, finite deformation theory, nonlinear partial differential equation, analytical solutions, canonical duality theory

\section{Nonconvex Variational Problem and Motivation}

A large class of finite deformation problems in nonlinear elasticity can be formulated on the basis of a variational principle $(\mathcal{P})$ in which it is required to minimize certain nonconvex potential energy. Typically, this takes the form

$$
(\mathcal{P}): \min _{\boldsymbol{\chi} \in \mathcal{X}_{a}}\left\{\Pi(\boldsymbol{\chi})=\int_{\Omega} W(\nabla \boldsymbol{\chi}) \mathrm{d} \Omega+\int_{\Omega} \phi(\boldsymbol{\chi}) \rho \mathrm{d} \Omega-\int_{\Gamma_{t}} \boldsymbol{\chi} \cdot \mathbf{t} \mathrm{d} \Gamma\right\},
$$


where $\chi$ represents the deformation field (a bijection), $W(\mathbf{F})$ is the strain energy per unit reference volume, which is a nonlinear differentiable function of the deformation gradient $\mathbf{F}=\nabla \boldsymbol{\chi}$, and $\nabla$ is the gradient operator in a simply-connected domain (the reference configuration of the body) $\Omega \subset \mathbb{R}^{3}$ with boundary $\partial \Omega=\Gamma=\Gamma_{t} \cup \Gamma_{\chi}$ such that $\Gamma_{t} \cap \Gamma_{\chi}=\emptyset$. Each material point in $\Omega$ is labeled by its position vector $\mathbf{X}$ and the corresponding point in the deformed configuration is denoted by $\mathbf{x}(=\chi(\mathbf{X}))$. The body force $\mathbf{f}$ (per unit mass) is taken to be conservative with potential $\phi(\mathbf{x})$ so that $\mathbf{f}=-\operatorname{grad} \phi$, and $\rho$ is the reference mass density. On the part $\Gamma_{t}$ of the boundary the surface traction $\mathbf{t}$ is prescribed to be of dead-load type, while on $\Gamma_{\chi}$ the deformation $\chi$ is given. The notation $\mathcal{X}_{a}$ identifies a kinematically admissible space of deformations $\chi$, defined by

$$
\mathcal{X}_{a}=\left\{\chi \in \mathcal{W}^{1, p}\left(\Omega ; \mathbb{R}^{3}\right) \mid \nabla \chi \in \mathcal{F}_{a}, \quad \chi=\chi_{0} \quad \text { on } \Gamma_{\chi}\right\}
$$

where $\mathcal{W}^{1, p}$ is the Sobolev space, i.e. a function space in which both $\chi$ and its weak derivative $\nabla \boldsymbol{\chi}$ have a finite $L^{p}(\Omega)$ norm. $\mathcal{F}_{a}=\left\{\mathbf{F} \in \mathcal{L}^{p}\left(\Omega ; \mathbb{R}^{3 \times 3}\right) \mid \operatorname{det} \mathbf{F}>0\right\}$ denotes the admissible deformation gradient space with $p>1$. Clearly, solutions $\chi \in \mathcal{X}_{a}$ of the problem $(\mathcal{P})$ are not necessarily to be smooth.

The criticality condition $\delta \Pi(\chi)=0$ leads to a mixed boundary-value problem $(B V P)$, namely

$$
(B V P):\left\{\begin{array}{l}
\nabla \cdot\left[\nabla_{\mathbf{F}} W(\nabla \boldsymbol{\chi})\right]+\rho \mathbf{f}=\mathbf{0} \quad \text { in } \boldsymbol{\Omega}, \\
\mathbf{n} \cdot\left[\nabla_{\mathbf{F}} W(\nabla \boldsymbol{\chi})\right]=\mathbf{t} \quad \text { on } \Gamma_{t},
\end{array}\right.
$$

where $\nabla_{\mathbf{F}} W(\nabla \boldsymbol{\chi})=\partial W(\mathbf{F}) / \partial \mathbf{F}$ (in components $\partial W / \partial F_{i \alpha}$ ), $\mathbf{n}$ is the unit outward normal to $\Gamma_{t}$ and, in component form, we adopt the conventions $\nabla \cdot \boldsymbol{\tau}=\left\{\partial \tau_{i \alpha} / \partial X_{\alpha}\right\}$ and $\boldsymbol{\tau} \cdot \mathbf{n}=$ $\left\{\tau_{i \alpha} n_{\alpha}\right\}$. Note that $\nabla \cdot \boldsymbol{\tau}$ is defined in the weak sense where $\nabla \boldsymbol{\chi}$ is discontinuous. In general, it is rarely possible to solve this nonlinear boundary-value problem by use of direct methods. Indeed, the strain energy $W(\mathbf{F})$ is a nonconvex function of $\mathbf{F}$, the problems $(\mathcal{P})$ and $(B V P)$ are not equivalent, and $(B V P)$ may possess multiple solutions. Identification of the global minimizer of the variational problem $(\mathcal{P})$ is a fundamentally difficult task in nonconvex analysis. From the point of view of numerical analysis, any numerical discretization of the problem $(\mathcal{P})$ leads to a nonconvex minimization problem, and it is well known in global optimization theory that most nonconvex minimization problems are NP-hard [14, 15, 16].

Duality principles play fundamental roles in sciences and engineering, especially in continuum mechanics and variational analysis. For linear elasticity, since the stored strain 
energy $W$ is a convex function of the (infinitesimal) strain tensor, it is well-known that each potential variational (primal) problem is linked a unique equivalent (dual) complementary variational problem via the conventional Legendre transformation. This one-to-one duality relation is also known as the complementary variational principle, which has been well-studied with extensive applications in both mathematical physics and engineering mechanics (see Arthurs, Nobel-Sewell, Oden-Reddy, Tabarrok-Rimrott, etc).

In finite deformation theory, if the stored energy density $W(\mathbf{F})$ is a strictly convex function of the deformation gradient tensor $\mathbf{F}$ over the field $\Omega$, then the first Piola-Kirchhoff stress tensor can be uniquely determined by $\boldsymbol{\tau}=\nabla W(\mathbf{F})$ and the complementary energy density $W^{*}$ can be obtained explicitly by the Legendre transformation:

$$
W^{*}(\boldsymbol{\tau})=\{\mathbf{F}: \boldsymbol{\tau}-W(\mathbf{F}) \mid \boldsymbol{\tau}=\nabla W(\mathbf{F})\},
$$

where $\mathbf{F}: \boldsymbol{\tau}$ is defined as $\operatorname{tr}\left(\mathbf{F} \cdot \boldsymbol{\tau}^{\mathrm{T}}\right)$ and ${ }^{\mathrm{T}}$ signifies the transpose. In this case, the complementary variational problem can be defined as

$$
\min _{\boldsymbol{\tau} \in \mathcal{T}_{a}}\left\{\Pi^{c}(\boldsymbol{\tau})=\int_{\Omega} W^{*}(\boldsymbol{\tau}) \mathrm{d} \Omega-\int_{\Gamma_{\chi}} \chi_{0} \cdot \boldsymbol{\tau} \cdot \mathbf{n} \mathrm{d} \Gamma\right\},
$$

where $\mathcal{T}_{a}$ is the statically admissible space defined by

$$
\mathcal{T}_{a}=\left\{\boldsymbol{\tau} \in \mathcal{L}^{q}(\Omega) \mid \nabla \cdot \boldsymbol{\tau}+\rho \mathbf{f}=\mathbf{0} \text { in } \boldsymbol{\Omega}, \boldsymbol{\tau} \cdot \mathbf{n}=\mathbf{t} \text { on } \boldsymbol{\Gamma}_{\mathbf{t}}\right\}
$$

where $q$ is the conjugate number of $p$, i.e. it is given by $1 / p+1 / q=1$. This complementary variational problem was first studied by Levinson [31]. The well-known Levinson principle states that if $\overline{\boldsymbol{\tau}}$ is a solution of the complementary variational problem (5), then the deformation field $\bar{\chi}$ defined through the inverse constitutive law $\mathbf{F}(\bar{\chi})=\nabla W^{*}(\overline{\boldsymbol{\tau}})$ is a solution of the potential variational problem (1) and the complementarity condition

$$
\Pi(\bar{\chi})+\Pi^{c}(\overline{\boldsymbol{\tau}})=0
$$

holds. This principle can be proved easily by using the traditional Lagrangian duality theory (see Gao, 2000).

The Levinson principle is simply the counterpart in finite deformation theory of the complementary variational principle in linear elasticity. In finite deformation theory, the stored strain energy $W(\mathbf{F})$ is in general nonconvex such that the stress-deformation relation $\boldsymbol{\tau}=\nabla W(\mathbf{F})$ is not uniquely invertible $[36,37]$ and the complementary energy function $W^{*}$ 
cannot be defined explicitly via the Legendre transformation. Although by the Fenchel transformation

$$
W^{\sharp}(\boldsymbol{\tau})=\max _{\mathbf{F}}\{\mathbf{F}: \boldsymbol{\tau}-W(\mathbf{F})\},
$$

the Fenchel-Moreau type dual problem can be formulated in the form of

$$
\max _{\boldsymbol{\tau} \in \mathcal{T}_{a}}\left\{\Pi^{\sharp}(\tau)=\int_{\Gamma_{\chi}} \chi_{0} \cdot \boldsymbol{\tau} \cdot \mathbf{n} \mathrm{d} \Gamma-\int_{\Omega} W^{\sharp}(\boldsymbol{\tau}) \mathrm{d} \Omega\right\},
$$

the nonconvexity of $W$ leads only to the so-called weak duality theorem

$$
\min _{\boldsymbol{\chi} \in \mathcal{X}_{a}} \Pi(\chi) \geq \max _{\boldsymbol{\tau} \in \mathcal{T}_{a}} \Pi^{\sharp}(\tau)
$$

due to the Fenchel-Young inequality $W(\mathbf{F}) \geq \mathbf{F}: \boldsymbol{\tau}-W^{\sharp}(\boldsymbol{\tau})$. In nonconvex analysis, the nonzero $\theta=\min {\boldsymbol{\chi} \in \mathcal{X}_{a}} \Pi(\boldsymbol{\chi})-\max _{\boldsymbol{\tau} \in \mathcal{T}_{a}} \Pi^{\sharp}(\boldsymbol{\tau})>0$ is called the duality gap. This duality gap shows that the well-developed Fenchel-Moreau duality theory can be used to solve mainly convex problems.

In finite deformation theory, the well-known Hellinger-Reissner principle $[25,38]$ and the Fraeijs de Veubeke principle [46] hold for both convex and nonconvex problems. However, these principles are not considered as pure complementary variational principles since the Hellinger-Reissner principle involves both the displacement field and the second PiolaKirchhoff stress tensor; while the Fraeijs de Veubeke principle has both the rotation tensor and the first Piola-Kirchhoff stress as its variational arguments. The existence of a pure complementary variational principle in general finite deformation theory has been discussed by many researchers over several decades (see, for example, [28, 29, 30, 32, 35, 36, 37]). Moreover, since the extremality condition in nonconvex variational analysis and global optimization is fundamentally difficult to resolve, none of the classical complementary-dual variational principles in finite deformation theory can be used for reliable numerical computations.

Canonical duality theory provides a potentially useful methodology for solving a large class of nonconvex problems in complex systems. This theory consists mainly of (1) a canonical dual transformation, which can be used to formulate perfect dual problems in nonconvex systems; (2) a complementary-dual variational principle, which allows a unified analytical solution form in terms of the canonical dual solutions; (3) a triality theory, which provides sufficient criteria for identifying both global and local extrema. The original idea 
of the canonical dual transformation was introduced by Gao and Strang [23] in finite deformation systems. In order to recover the duality gap in nonconvex variational problems, they discovered a so-called complementary gap function, which leads to a complementary-dual variational principle in finite deformation mechanics. They proved that if this gap function is positive on a dual feasible space, the generalized Hellinger-Reissner energy is a saddlefunctional. It turns out that this gap function provides a sufficient condition for global optimal solution in nonconvex variational problems. Seven years later, it was realized that the negative gap function can be used to identify local extrema. Therefore, a triality theory was first proposed in post-buckling problems of a large deformation beam model [7], and a pure complementary energy principle was eventually obtained in [9]. This principle can be used to obtain a general analytical solution for 3-D large deformation elasto-plasticity [11]. It was shown by Gao and Ogden (see $[19,20]$ ) that for one-dimensional nonlinear elasticity problems, both global and local minimal solutions are usually nonsmooth and can't be obtained by any Newton type of numerical methods. For finite dimensional systems, the canonical duality theory has been successfully applied for solving a large class of challenging problems in computational mechanics $[4,24,43]$ and global optimization with extensive applications in computational biology [47], chaotic dynamical systems [33, 41], discrete and network optimization [18, 21, 40, 42].

The purpose of this paper is to illustrate the application of the pure complementary variational principle in combination with triality theory by solving a general nonconvex variational problem governed by St Venant-Kirchhoff material. The paper is organized as follows. Section 2 presents a brief review on the canonical duality theory in nonlinear elasticity. Some fundamental issues in nonlinear elasticity are addressed, including the reasons why the Legendre-Hadamard condition provides only necessary condition for local minima, how the Gao-Strang gap function and the triality theory can be used to identify both global and local extremal solutions. In Section 3 we show that for the St VenantKirchhoff materials, the pure complementary variational problem can be solved principally to obtain all possible solutions. Some concluding remarks are contained in Section 4 . 


\section{Canonical Duality Theory and Complementary Variational Principle}

It is known that the stored-energy function $W: \mathcal{F}_{a} \rightarrow \mathbb{R}$ must obey certain physical laws and requirements in continuum mechanics, such as the principle of material frame-indifference [45], which lay a mathematical foundation for the canonical duality theory. Let

$$
\mathrm{SO}(3)=\left\{\mathbf{Q} \in \mathbb{R}^{3 \times 3} \mid \mathbf{Q}^{T}=\mathbf{Q}^{-1}, \operatorname{det} \mathbf{Q}=1\right\}
$$

be the special orthogonal group.

\section{Definition 2.1 (Objectivity and Isotropy [12])}

(D1) Objective Set and Objective Function: A subset $\mathcal{F}_{a} \subset \mathbb{R}^{3 \times 3}$ is said to be objective if for every $\mathbf{F} \in \mathcal{F}_{a}$ and every $\mathbf{Q} \in \mathrm{SO}(3), \mathbf{Q F} \in \mathcal{F}_{a}$. A scalarvalued function $W: \mathcal{F}_{a} \rightarrow \mathbb{R}$ is said to be objective if its domain is objective and

$$
W(\mathbf{Q F})=W(\mathbf{F}) \quad \forall \mathbf{F} \in \mathcal{F}_{a}, \forall \mathbf{Q} \in \mathrm{SO}(3)
$$

(D2) Isotropic Set and Isotropic Function: A subset $\mathcal{F}_{a} \subset \mathbb{R}^{3 \times 3}$ is said to be isotropic if for every $\mathbf{F} \in \mathcal{F}_{a}$ and every $\mathbf{Q} \in \mathrm{SO}(3), \mathbf{F Q} \in \mathcal{F}_{a}$. A scalarvalued function $W: \mathcal{F}_{a} \rightarrow \mathbb{R}$ is said to be isotropic if its domain is isotropic and

$$
W(\mathbf{F Q})=W(\mathbf{F}) \quad \forall \mathbf{F} \in \mathcal{F}_{a}, \forall \mathbf{Q} \in \mathrm{SO}(3)
$$

The objectivity implies that the constitutive law of material is independent with the observer (coordinate free). While the isotropy means that the material possesses certain symmetry. Generally speaking, the deformation gradient $\mathbf{F}$ is a two-point tensor, which is not considered as a strain measure. The right Cauchy-Green tensor $\mathbf{C}=\mathbf{F}^{T} \mathbf{F}$ is a (Lagrange type) strain measure which is objective (rotation free), i.e.,

$$
\mathbf{C}(\mathbf{Q F})=(\mathbf{Q F})^{T}(\mathbf{Q F})=\mathbf{F}^{T} \mathbf{Q}^{T} \mathbf{Q F}=\mathbf{C}(\mathbf{F}) \forall \mathbf{Q} \in \mathrm{SO}(3)
$$

Dually, the left Cauchy-Green tensor $\mathbf{B}=\mathbf{F}^{T} \mathbf{F}$ is an isotropic function of $\mathbf{F}$.

In continuum mechanics, the objectivity is also known as the principle of frame-indifference. According to P.G. Ciarlet, the stored energy function of a hyper-elastic material is objective 
if and only if there exists a function $U(\mathbf{C})$ such that $W(\mathbf{F})=U(\mathbf{C}(\mathbf{F})$ ) (see Theorem 4.2-1 in [5]). This principle lays a foundation for the canonical duality theory.

Indeed, the canonical dual transformation was developed from the concept of the objectivity. The key step of this transformation is the introduction of a geometrically admissible strain measure $\boldsymbol{\xi}=\boldsymbol{\Lambda}(\chi): \mathcal{X}_{a} \rightarrow \mathcal{E}_{a} \subset \mathbb{R}^{3 \times 3}$ and the canonical function $U(\boldsymbol{\xi}): \mathcal{E}_{a} \rightarrow \mathbb{R}$ such that the nonconvex stored energy $W(\mathbf{F})$ can be written in the canonical form $W(\nabla \chi)=$ $U(\boldsymbol{\Lambda}(\boldsymbol{\chi}))$. According to [12], a convex differentiable real-valued function $U(\boldsymbol{\xi})$ is said to be canonical on its domain $\mathcal{E}_{a}$ if the duality relation $\boldsymbol{\xi}^{*}=\nabla U(\boldsymbol{\xi}): \mathcal{E}_{a} \rightarrow \mathcal{E}_{a}^{*}$ is invertible such that the conjugate function $U^{*}\left(\boldsymbol{\xi}^{*}\right)$ of $U(\boldsymbol{\xi})$ can be defined uniquely by the Legendre transformation

$$
U^{*}\left(\boldsymbol{\xi}^{*}\right)=\left\{\boldsymbol{\xi}: \boldsymbol{\xi}^{*}-U(\boldsymbol{\xi}) \mid \boldsymbol{\xi}^{*}=\nabla U(\boldsymbol{\xi}) \forall \boldsymbol{\xi} \in \mathcal{E}_{a}\right\}
$$

By the theory of convex analysis, it is easy to prove that the following canonical duality relations hold on $\mathcal{E}_{a} \times \mathcal{E}_{a}^{*}$

$$
\boldsymbol{\xi}^{*}=\nabla U(\boldsymbol{\xi}) \Leftrightarrow \boldsymbol{\xi}=\nabla U^{*}\left(\boldsymbol{\xi}^{*}\right) \Leftrightarrow U(\boldsymbol{\xi})+U^{*}\left(\boldsymbol{\xi}^{*}\right)=\boldsymbol{\xi}: \boldsymbol{\xi}^{*}
$$

and the pair $\left(\boldsymbol{\xi}, \boldsymbol{\xi}^{*}\right)$ is called the canonical dual pair on $\mathcal{E}_{a} \times \mathcal{E}_{a}^{*}$.

Thus, on replacing $W(\nabla \chi)$ in the total potential energy $\Pi(\chi)$ by its canonical form $W(\nabla \boldsymbol{\chi})=U(\boldsymbol{\Lambda}(\boldsymbol{\chi}))$, and we take the body force to be a constant, so that $\phi(\boldsymbol{\chi})=-\mathbf{f} \cdot \boldsymbol{\chi}$, the minimal potential energy variational problem (1) can be written in the following canonical form

$$
(\mathcal{P}): \min _{\boldsymbol{\chi} \in \mathcal{X}_{a}^{\prime}}\left\{\Pi(\boldsymbol{\chi})=\int_{\Omega}[U(\boldsymbol{\Lambda}(\boldsymbol{\chi}))-\rho \boldsymbol{\chi} \cdot \mathbf{f}] \mathrm{d} \boldsymbol{\Omega}-\int_{\boldsymbol{\Gamma}_{\mathbf{t}}} \boldsymbol{\chi} \cdot \mathbf{t} \mathrm{d} \boldsymbol{\Gamma}\right\} .
$$

Furthermore, in terms of $\varsigma=\boldsymbol{\xi}^{*}$ and by the Fenchel-Young equality

$$
U(\boldsymbol{\Lambda}(\chi))=\boldsymbol{\Lambda}(\chi): \varsigma-U^{*}(\varsigma)
$$

the so-called total complementary energy functional [23] $\Xi: \mathcal{X}_{a} \times \mathcal{E}_{a}^{*} \rightarrow \mathbb{R}$ can be written, in the present context, as

$$
\Xi(\chi, \varsigma)=\int_{\Omega}\left[\boldsymbol{\Lambda}(\chi): \varsigma-U^{*}(\boldsymbol{\varsigma})-\rho \boldsymbol{\chi} \cdot \mathbf{f}\right] \mathrm{d} \Omega-\int_{\Gamma_{t}} \boldsymbol{\chi} \cdot \mathbf{t} \mathrm{d} \Gamma .
$$

For a given statically admissible field $\boldsymbol{\tau} \in \mathcal{T}_{a}$, this total complementary functional can be written in the following form

$$
\Xi_{\boldsymbol{\tau}}(\boldsymbol{\chi}, \boldsymbol{\varsigma})=\int_{\Gamma_{\chi}} \boldsymbol{\chi}_{0} \cdot \boldsymbol{\tau} \cdot \mathbf{n} \mathrm{d} \Gamma+\int_{\Omega}\left[\boldsymbol{\Lambda}(\boldsymbol{\chi}): \varsigma-U^{*}(\boldsymbol{\varsigma})-(\nabla \boldsymbol{\chi}): \boldsymbol{\tau}\right] \mathrm{d} \Omega .
$$


For a given $\varsigma \in \mathcal{E}_{a}^{*}$, the canonical dual functional $\Pi^{d}(\varsigma)$ is then defined by

$$
\Pi^{d}(\varsigma)=\{\Xi(\chi, \varsigma) \mid \delta \chi \Xi(\chi, \varsigma)=0\}=F^{\Lambda}(\varsigma)-\int_{\Omega} U^{*}(\varsigma) \mathrm{d} \Omega,
$$

where $F^{\boldsymbol{\Lambda}}(\boldsymbol{\varsigma})$ is defined by the so-called $\boldsymbol{\Lambda}$-conjugate transformation $[12,16]$

$$
F^{\boldsymbol{\Lambda}}(\boldsymbol{\varsigma})=\operatorname{sta}\left\{\int_{\Omega}[\boldsymbol{\Lambda}(\chi): \varsigma-\rho \chi \cdot \mathbf{f}] \mathrm{d} \boldsymbol{\Omega}-\int_{\Gamma_{\mathbf{t}}} \chi \cdot \mathbf{t d} \boldsymbol{\Gamma} \mid \chi \in \mathcal{X}_{\mathbf{a}}\right\},
$$

with sta indicating the stationary value at fixed $\varsigma \in \mathcal{E}_{a}^{*}$. In terms of $\tau \in \mathcal{T}_{a}$, we have the following form

$$
F_{\boldsymbol{\tau}}^{\boldsymbol{\Lambda}}(\boldsymbol{\varsigma})=\int_{\Gamma_{\chi}} \boldsymbol{\chi}_{0} \cdot \boldsymbol{\tau} \cdot \mathbf{n} \mathrm{d} \Gamma+\operatorname{sta}\left\{\int_{\Omega}[\boldsymbol{\Lambda}(\boldsymbol{\chi}): \varsigma-(\nabla \boldsymbol{\chi}): \boldsymbol{\tau}] \mathrm{d} \Omega \mid \chi \chi \mathcal{X}_{a}\right\} .
$$

In finite deformation theory,

$$
\Pi_{\boldsymbol{\tau}}^{d}(\boldsymbol{\varsigma})=F_{\boldsymbol{\tau}}^{\boldsymbol{\Lambda}}(\boldsymbol{\varsigma})-\int_{\Omega} U^{*}(\boldsymbol{\varsigma}) \mathrm{d} \Omega
$$

is also called the pure complementary energy functional, which was first proposed in [9].

Theorem 2.1 (Complementary-Dual Variational Principle [11]) For a given statically admissible field $\boldsymbol{\tau} \in \mathcal{T}_{a}$, the following statements are equivalent:

1. $(\bar{\chi}, \overline{\boldsymbol{\varsigma}})$ is a critical point of $\Xi_{\boldsymbol{\tau}}(\boldsymbol{\chi}, \boldsymbol{\varsigma})$;

2. $\bar{\chi}$ is a critical point of $\Pi(\chi)$;

3. $\overline{\boldsymbol{\varsigma}}$ is a critical point of $\Pi_{\boldsymbol{\tau}}^{d}(\boldsymbol{\varsigma})$.

Moreover, we have

$$
\Pi(\bar{\chi})=\Xi(\bar{\chi}, \bar{\varsigma})=\Xi_{\boldsymbol{\tau}}(\bar{\chi}, \overline{\boldsymbol{\varsigma}})=\Pi_{\boldsymbol{\tau}}^{d}(\overline{\boldsymbol{\varsigma}})
$$

This theorem shows that to find a critical solution to the nonconvex total potential $\Pi(\chi)$ is equivalent to find a critical point of its canonical dual function $\Pi_{\boldsymbol{\tau}}^{d}(\varsigma)$. For a given $\boldsymbol{\tau} \in \mathcal{T}_{a}$, different choice of the geometrical measure $\boldsymbol{\Lambda}(\chi)$ will leads to different, but equivalent, $\Pi_{\mathcal{\tau}}^{d}(\varsigma)$ on a subset $\mathcal{S}_{a} \subset \mathcal{E}_{a}^{*}$.

In finite deformation theory, the canonical duality relation is also known as the Hill work conjugate and the canonical function $U(\boldsymbol{\xi})$ is called strain energy-density. According to Hill, for a given hyper-elastic material, there exist a class of strain measures $\boldsymbol{\xi}$ and the 
associated canonical functions $U(\boldsymbol{\xi})$ such that the associated stress can by defined uniquely by the canonical duality relation $\boldsymbol{\xi}^{*}=\nabla U(\boldsymbol{\xi})$. There are many canonical strain measures in finite elasticity and many of these strain measures belong to the well-known Hill-Seth strain family

$$
\mathbf{E}^{(\eta)}=\frac{1}{2 \eta}\left[\mathbf{C}^{\eta}-\mathbf{I}\right]
$$

where $\mathbf{I}$ is an identity tensor in $\mathbb{R}^{3 \times 3}$ and $\eta$ is a real number.

Canonical duality theory and pure complementary energy principle for general strain measures have been studied in [12]. In this paper, we consider only the Green-St Venant strain tensor $\mathbf{E}^{(1)}$, simply denoted as $\mathbf{E}$. In this case, the geometrical operator

$$
\mathbf{E}=\Lambda(\chi)=\frac{1}{2}\left[(\nabla \boldsymbol{\chi})^{T}(\nabla \boldsymbol{\chi})-\mathbf{I}\right]: \mathcal{X}_{a} \rightarrow \mathcal{E}_{a}
$$

is a quadratic operator and its domain can be defined by

$$
\mathcal{E}_{a}=\left\{\mathbf{E} \in \mathcal{L}^{p / 2}\left(\Omega ; \mathbb{R}^{3 \times 3}\right) \mid \mathbf{E}=\mathbf{E}^{T},(2 \mathbf{E}+\mathbf{I}) \succ 0\right\}
$$

We assume that the associated strain energy density $U(\mathbf{E}): \mathcal{E}_{a} \rightarrow \mathbb{R}$ is convex such that the conjugate stress $\boldsymbol{\varsigma}$ of $\mathbf{E}$, denoted by $\mathbf{S}$, can be defined uniquely by the constitutive law

$$
\mathbf{S}=\nabla U(\mathbf{E}): \mathcal{E}_{a} \rightarrow \mathcal{E}_{a}^{*}
$$

This associated stress $\mathbf{S}$ is the well-known second Piola-Kirchhoff stress, which is welldefined on $\mathcal{E}_{a}^{*}=\left\{\mathbf{S} \in \mathcal{L}^{p /(p-2)}\left(\Omega ; \mathbb{R}^{3 \times 3}\right) \mid \mathbf{S}=\mathbf{S}^{T}\right\}$. In this case, the pure complementary energy $\Pi_{\mathcal{\tau}}^{d}$ has the form of

$$
\Pi_{\boldsymbol{\tau}}^{d}(\mathbf{S})=\int_{\Gamma_{\chi}} \boldsymbol{\chi}_{0} \cdot \boldsymbol{\tau} \cdot \mathbf{n} \mathrm{d} \Gamma-\int_{\Omega}\left[\frac{1}{2} \operatorname{tr}\left(\boldsymbol{\tau} \cdot \mathbf{S}^{-1} \cdot \boldsymbol{\tau}+\mathbf{S}\right)+U^{*}(\mathbf{S})\right] \mathrm{d} \Omega
$$

which is well-defined on the canonical dual space

$$
\mathcal{S}_{a}=\left\{\mathbf{S} \in \mathcal{E}_{a}^{*} \mid \operatorname{tr}\left(\boldsymbol{\tau} \cdot \mathbf{S}^{-1} \cdot \boldsymbol{\tau}\right) \in \mathcal{L}^{1}(\Omega ; \mathbb{R}) \forall \boldsymbol{\tau} \in \mathcal{T}_{a}\right\}
$$

Therefore, the canonical dual problem is to find the critical point $\overline{\mathbf{S}} \in \mathcal{S}_{a}$ such that

$$
\left(\mathcal{P}^{d}\right): \Pi_{\boldsymbol{\tau}}^{d}(\overline{\mathbf{S}})=\operatorname{sta}\left\{\Pi_{\boldsymbol{\tau}}^{d}(\mathbf{S}) \mid \mathbf{S} \in \mathcal{S}_{a}\right\} .
$$

Theorem 2.2 (Analytical Solution Form [12]) For a given $\boldsymbol{\tau} \in \mathcal{T}_{a}$, if $\overline{\mathbf{S}}$ is a critical point of $\Pi_{\boldsymbol{\tau}}^{d}(\mathbf{S})$, then along any path from $\mathbf{X}_{\mathbf{0}} \in \boldsymbol{\Gamma}_{\chi}$ to $\mathbf{X} \in \boldsymbol{\Omega}$, the deformation defined by

$$
\bar{\chi}=\int_{\mathbf{X}_{0}}^{\mathbf{X}} \boldsymbol{\tau} \cdot \overline{\mathbf{S}}^{-1} \cdot \mathrm{d} \mathbf{X}+\chi_{\mathbf{0}}\left(\mathbf{X}_{\mathbf{0}}\right)
$$


is a critical solution to $(\mathcal{P})$. Moreover, if

$$
\nabla \times\left(\boldsymbol{\tau} \cdot \overline{\mathbf{S}}^{-1}\right)=\mathbf{0}
$$

then $\bar{\chi}$ is a closed form solution to the boundary value problem (BVP) (3).

The proof of this theorem can be found in $[8,9,11]$. In fact, the criticality condition $\delta \Pi_{\boldsymbol{\tau}}^{d}(\mathbf{S})=0$ leads to the following dual tensor equation:

$$
\mathbf{S} \cdot\left[\mathbf{I}+2\left(\nabla U^{*}(\mathbf{S})\right)\right] \cdot \mathbf{S}=\boldsymbol{\tau}^{\mathrm{T}} \cdot \boldsymbol{\tau}
$$

which is equivalent to

$$
\nabla U^{*}(\overline{\mathbf{S}})=\frac{1}{2}\left(\left(\boldsymbol{\tau} \cdot \overline{\mathbf{S}}^{-1}\right)^{T} \boldsymbol{\tau} \cdot \overline{\mathbf{S}}^{-1}-\mathbf{I}\right) .
$$

This is actually the constitutive law $\mathbf{E}=\boldsymbol{\Lambda}(\overline{\boldsymbol{\chi}})=\frac{1}{2}\left[\mathbf{F}^{T} \mathbf{F}-\mathbf{I}\right]=\nabla U^{*}(\overline{\mathbf{S}})$ subjected to $\mathbf{F}=\boldsymbol{\tau} \cdot \overline{\mathbf{S}}^{-1}$. Therefore, if the compatibility condition $\nabla \times \mathbf{F}=\mathbf{0}$, in index notation

$$
\frac{\partial F_{i \alpha}}{\partial X_{\beta}}=\frac{\partial F_{i \beta}}{\partial X_{\alpha}}
$$

holds, then $\mathbf{F}$ is the deformation gradient and $\bar{\chi}$ is a solution to $(B V P)$.

Remark 2.1 (PDE $\Leftrightarrow$ Algebraic Equation) Theorem 2.2 shows that by the pure complementary energy principle, the nonlinear partial differential equation $(B V P)$ is equivalently converted to a canonical dual tensor equation (29), which can be solved to obtain the stress field $\overline{\mathbf{S}}$ for certain materials. From the equation (29) we know that $\mathbf{S}=\mathbf{0}$ if $\boldsymbol{\tau}=\mathbf{0}$. Therefore, although $\mathbf{S}^{-1}$ appears in $\Pi_{\boldsymbol{\tau}}^{d}(\mathbf{S})$, this pure complementary energy is well-defined on $\mathcal{S}_{a}$. The equation (27) presents an analytical solution form to the boundary value problem in terms of the canonical dual stress field $\overline{\mathbf{S}}$ and the statically admissible $\boldsymbol{\tau} \in \mathcal{T}_{a}$. Of course, this is purely formal and in general it is not easy to obtain the solution for general practices unless the deformation compatibility condition (28) holds.

It has been assumed here that the relation between $\mathbf{S}$ and $\mathbf{E}$ is invertible. This certainly holds in a neighborhood of the (stress-free) reference configuration since the canonical strain energy $U(\mathbf{E})$ is convex in such a neighborhood. It is a reasonable assumption to extend this to a sufficiently large domain that includes deformations of practical interest. Finite element implementations of nonlinear elasticity are usually based on the variables $\mathbf{S}$ and $\mathbf{E}$ and the associated tangent tensor $\partial \mathbf{S} / \partial \mathbf{E}=\nabla^{2} U(\mathbf{E})$, which is assumed to be positive definite. It is always possible to select forms of the strain-energy function $W$ such that this 
is the case, although the possibility of its failure for particular materials is not in general ruled out.

In terms of the deformation $\chi \in \mathcal{X}_{a}$ and the second Piola-Kirchhoff stress $\mathbf{S} \in \mathcal{E}_{a}^{*}$, the total complementary functional $\Xi(\chi, \mathbf{S})$ can be written as

$$
\Xi_{\boldsymbol{\tau}}(\boldsymbol{\chi}, \mathbf{S})=\int_{\Omega}\left[\mathbf{E}(\boldsymbol{\chi}): \mathbf{S}-U^{*}(\mathbf{S})-(\nabla \boldsymbol{\chi}): \boldsymbol{\tau}\right] \mathrm{d} \Omega+\int_{\Gamma_{\chi}} \boldsymbol{\chi}_{0} \cdot \boldsymbol{\tau} \cdot \mathbf{n} \mathrm{d} \Gamma
$$

which is actually the well-known Hellinger-Reissner energy if the first Piola-Kirchhoff stress is replaced by external force field. From the nonlinear canonical dual tensor equation (29) we know that for a given $\boldsymbol{\tau} \in \mathcal{T}_{a}$, the pure complementary energy $\Pi_{\boldsymbol{\tau}}^{d}(\mathbf{S})$ may have multiple critical points. In order to identify the global extremum, We need to introduce the following subspaces:

$$
\mathcal{S}_{a}^{+}=\left\{\mathbf{S} \in \mathcal{S}_{a} \mid \mathbf{S} \succ 0\right\}, \mathcal{S}_{a}^{-}=\left\{\mathbf{S} \in \mathcal{S}_{a} \mid \mathbf{S} \prec 0\right\}
$$

Theorem 2.3 Suppose for a given $\boldsymbol{\tau} \in \mathcal{T}_{a}$, the pair $(\overline{\boldsymbol{\chi}}, \overline{\mathbf{S}})$ is an isolated critical point of $\Xi_{\boldsymbol{\tau}}(\chi, \mathbf{S})$. If $\overline{\mathbf{S}} \in \mathcal{S}_{a}^{+}$, then $\overline{\boldsymbol{\chi}}$ is a global minimizer of $\Pi(\chi)$ on $\mathcal{X}_{a}$ if and only if $\overline{\mathbf{S}}$ is a global maximizer of $\Pi_{\boldsymbol{\tau}}^{d}(\mathbf{S})$ on $\mathcal{S}_{a}^{+}$, i.e.,

$$
\Pi(\overline{\boldsymbol{\chi}})=\min _{\boldsymbol{\chi} \in \mathcal{X}_{a}} \Pi(\chi) \Leftrightarrow \max _{\mathbf{S} \in \mathcal{S}_{a}^{+}} \Pi_{\boldsymbol{\tau}}^{d}(\mathbf{S})=\Pi_{\boldsymbol{\tau}}^{d}(\overline{\mathbf{S}}) .
$$

If $\overline{\mathbf{S}} \in \mathcal{S}_{a}^{-}$, then $\bar{\chi}$ is a local maximizer of $\Pi(\chi)$ if and only if $\overline{\mathbf{S}}$ is a local maximizer of $\Pi_{\boldsymbol{\tau}}^{d}(\mathbf{S})$, i.e., on a neighborhood $\mathcal{X}_{o} \times \mathcal{S}_{o} \subset \mathcal{X}_{a} \times \mathcal{S}_{a}^{-}$,

$$
\Pi(\bar{\chi})=\max _{\chi \in \mathcal{X}_{o}} \Pi(\chi) \Leftrightarrow \max _{\mathbf{S} \in \mathcal{S}_{o}} \Pi_{\boldsymbol{\tau}}^{d}(\mathbf{S})=\Pi_{\boldsymbol{\tau}}^{d}(\overline{\mathbf{S}}) .
$$

If $\overline{\mathbf{S}} \in \mathcal{S}_{a}^{-}$and $\nabla_{\mathbf{F}}^{2} W(\nabla \overline{\boldsymbol{\chi}}) \succ 0$, then $\overline{\boldsymbol{\chi}}$ is a local minimizer of $\Pi(\boldsymbol{\chi})$.

\section{Remark 2.2 (The Complementary Gap Function and Triality Theory)}

Theorem 2.3 shows that the extremality of the primal solution $\chi$ depends on its canonical dual solution $\mathbf{S}$. This result was first discovered by Gao and Strang in 1989 [23], i.e. they proved that $\overline{\boldsymbol{\chi}}(\overline{\mathbf{S}})$ is a global minimizer of $\Pi(\chi)$ if the complementary gap function satisfies

$$
G_{a p}(\boldsymbol{\chi}, \overline{\mathbf{S}})=\int_{\Omega} \frac{1}{2}\left[(\nabla \boldsymbol{\chi})^{T}(\nabla \boldsymbol{\chi})+\mathbf{I}\right]: \overline{\mathbf{S}} d \Omega \geq 0 \forall \boldsymbol{\chi} \in \mathcal{X}_{a}
$$


Since $G_{a p}(\chi, \overline{\mathbf{S}})$ is quadratic in $\boldsymbol{\chi}$, this gap function is positive for any given $\chi \in \mathcal{X}_{a}$ if $\overline{\mathbf{S}} \succeq 0$. Replacing $\mathbf{F}=\nabla \chi$ by $\mathbf{F}=\boldsymbol{\tau} \cdot \mathbf{S}^{-1}$, this gap function can be written as the so-called pure gap function

$$
G_{a p}(\chi(\mathbf{S}), \mathbf{S})=\int_{\Omega} \frac{1}{2} \operatorname{tr}\left(\boldsymbol{\tau} \cdot \mathbf{S}^{-1} \cdot \boldsymbol{\tau}+\mathbf{S}\right) d \Omega,
$$

which is a main term in the pure complementary energy $\Pi_{\boldsymbol{\tau}}^{d}(\mathbf{S})$ in addition to $U^{*}(\mathbf{S})$. Comparing $\Pi_{\boldsymbol{\tau}}^{d}(\mathbf{S})$ with $\Pi^{\sharp}(\boldsymbol{\tau})$ given by $(7)$, we can understand that this gap function not only recovers the duality gap in the Fenchel-Moreau duality theory, but also provides a global extremality condition for nonconvex variational problem $(\mathcal{P})$.

To see this in detail, let us consider the canonical transformation $W(\mathbf{F})=U(\mathbf{E}(\mathbf{F}))$. By chain rule we have

$$
\frac{\partial^{2} W(\mathbf{F})}{\partial F_{\alpha}^{i} \partial F_{\beta}^{j}}=\delta^{i j} S_{\alpha \beta}+\sum_{\theta, \nu=1}^{3} F_{\theta}^{i} H_{\theta \alpha \beta \nu} F_{\nu}^{j},
$$

where $\mathbf{H}=\left\{H_{\theta \alpha \beta \nu}\right\}=\nabla^{2} U(\mathbf{E})$. By the convexity of the canonical function $U(\mathbf{E})$, we have $\mathbf{H} \succ 0$. Therefore, if $\mathbf{S}=\left\{S_{\alpha \beta}\right\} \in \mathcal{S}_{a}^{+}$, the Hessian $\nabla^{2} W(\mathbf{F}) \succ 0$ and, by Gao and Strang [23], the associated deformation field $\boldsymbol{\chi}$ is a global minimizer of $\Pi(\chi)$. The statement (32) shows that the nonconvex minimization problem $(\mathcal{P})$ is equivalent to a concave maximization dual problem over a convex space $\mathcal{S}_{a}^{+}$, i.e.,

$$
\max \left\{\Pi_{\boldsymbol{\tau}}^{d}(\mathbf{S}) \mid \mathbf{S} \in \mathcal{S}_{a}^{+}\right\}
$$

which is much easier than the nonconvex primal problem $(\mathcal{P})$. The global optimality condition $\mathbf{S} \in \mathcal{S}_{a}^{+}$is a strong case of Gao and Strang's positive gap function (34).

Subsequently, in a study of post-buckling analysis for a nonlinear beam theory, it was found that if the dual solution $\overline{\mathbf{S}}$ is negative definite in the domain $\Omega$, the solution $\bar{\chi}$ could be either a local minimizer or a local maximizer of the total potential energy. To see this, we substitutive $\mathbf{F}=\boldsymbol{\tau} \cdot \mathbf{S}^{-1}$ into (36) to obtain

$$
\frac{\partial^{2} W(\mathbf{F})}{\partial F_{\alpha}^{i} \partial F_{\beta}^{j}}=\delta^{i j} S_{\alpha \beta}+\sum_{\theta, \nu, \delta, \lambda=1}^{3} \tau_{\theta}^{i} S_{\theta \delta}^{-1} H_{\delta \alpha \beta \nu} S_{\nu \lambda}^{-1} \tau_{\lambda}^{j}
$$

which shows that even if $\mathbf{S} \prec 0$, the Hessian matrix $\nabla^{2} W(\mathbf{F})$ could be either positive or negative definite, depending on the eigenvalues of $\mathbf{S} \in \mathcal{S}_{a}^{-}$. Thus, in addition to the doublemax duality (33), we have the so-called double-min duality

$$
\Pi(\overline{\boldsymbol{\chi}})=\min _{\boldsymbol{\chi} \in \mathcal{X}_{o}} \Pi(\boldsymbol{\chi}) \Leftrightarrow \min _{\mathbf{S} \in \mathcal{S}_{o}} \Pi_{\boldsymbol{\tau}}^{d}(\mathbf{S})=\Pi_{\boldsymbol{\tau}}^{d}(\overline{\mathbf{S}}),
$$


which holds under certain condition (see [15]). For this reason, a so-called triality theory was proposed first in post-buckling analysis of a large deformed beam model [7], and then in general nonconvex mechanics [11, 12]. This triality theory reveals an important fact in nonconvex analysis, i.e. for a given statically admissible field $\boldsymbol{\tau} \in \mathcal{T}_{a}$, if the canonical dual equation (29) has multiple solutions $\left\{\mathbf{S}_{k}\right\}$ in a subset $\Omega_{o} \subset \Omega$, then the boundary value problem $(B V P)$ could have an infinite number of solutions $\left\{\chi_{k}(\mathbf{X})\right\}$ in $\Omega$. The wellknown Legendre-Hadamard $(L-H)$ condition is only a necessary condition for a local minimal solution, while the triality theory can identify not only the global minimizers, but also both local minimizers and local maximizers. It is known that an elliptic equation is corresponding to a convex variational problem. If the boundary-value problem (3) has multiple solutions $\left\{\chi_{k}(\mathbf{X})\right\}$ at one material point $\mathbf{X} \in \Omega$, the total potential $\Pi(\chi)$ is not convex and the operator $A(\boldsymbol{\chi})=\nabla \cdot\left[\nabla_{\mathbf{F}} W(\nabla \boldsymbol{\chi})\right]$ may not be elliptic at $\mathbf{X} \in \Omega$ even if the $L-H$ condition holds at certain $\chi_{k}(\mathbf{X})$.

The pure complementary energy principle and triality theory play a fundamental role not only in nonconvex analysis, but also in computational science and global optimization (see $[15,17,18,22])$.

\section{Application to St Venant-Kirchhoff Material}

For St. Venant-Kirchhoff material, the canonical energy function $U(\mathbf{E})$ has the most simple form:

$$
U(\mathbf{E})=\mu \operatorname{tr}\left(\mathbf{E}^{\mathbf{2}}\right)+\frac{\mathbf{1}}{\mathbf{2}} \lambda(\operatorname{tr} \mathbf{E})^{2} .
$$

The second Piola-Kirchhoff stress depends linearly on the Green-St Venant strain via the Hooke's law:

$$
\mathbf{S}=\nabla U(\mathbf{E})=2 \mu \mathbf{E}+\lambda(\operatorname{tr} \mathbf{E}) \mathbf{I}=\mathbf{H}: \mathbf{E},
$$

where $\mathbf{H}$ is the Hooke tensor for St Venant-Kirchhoff material. The complementary energy is

$$
U^{*}(\mathbf{S})=\frac{1}{4 \mu} \operatorname{tr}\left(\mathbf{S}^{2}\right)-\frac{\lambda}{4 \mu(3 \lambda+2 \mu)}(\operatorname{tr} \mathbf{S})^{2}
$$

and hence

$$
\mathbf{E}=\nabla U^{*}(\mathbf{S})=\frac{1}{2 \mu} \mathbf{S}-\frac{\lambda}{2 \mu(3 \lambda+2 \mu)}(\operatorname{tr} \mathbf{S}) \mathbf{I} \equiv \mathbf{H}^{-\mathbf{1}}: \mathbf{S} .
$$


By the canonical dual tensor equation (29), we have

$$
\mathbf{S}^{2}+2 \mathbf{S}\left(\mathbf{H}^{-1}: \mathbf{S}\right) \mathbf{S}=\mathbf{S}^{2}+\frac{1}{\mu} \mathbf{S}^{3}-\frac{\lambda}{\mu(3 \lambda+2 \mu)}(\operatorname{tr} \mathbf{S}) \mathbf{S}^{2}=\boldsymbol{\tau}^{T} \boldsymbol{\tau} .
$$

The diagonalization of this tensor equation leads to the following coupled quebec nonlinear algebraic systems:

$$
S_{i}^{2}+\frac{1}{\mu} S_{i}^{3}-\frac{\lambda}{\mu(3 \lambda+2 \mu)}\left(S_{1}+S_{2}+S_{3}\right) S_{i}^{2}=\tau_{i}^{2} \quad i=1,2,3 .
$$

For convenience, we make the following substitutions in (45):

$$
S_{i}=\mu \varsigma_{i}, \quad \tau_{i}^{2}=\mu^{2} \sigma_{i}, \quad i=1,2,3,
$$

and $k=\frac{\lambda}{3 \lambda+2 \mu}<1 / 3$ (due to $\mu>0$ ). So, the system (45) can be written as follows

$$
\varsigma_{i}^{3}+\varsigma_{i}^{2}-k\left(\varsigma_{1}+\varsigma_{2}+\varsigma_{3}\right) \varsigma_{i}^{2}=\sigma_{i}, \quad i=1,2,3
$$

\subsection{Auxiliary Equation}

In this section we will study solutions of the following equation:

$$
G(\varsigma, q, \sigma)=\varsigma^{3}+(1-k q) \varsigma^{2}-\sigma=0
$$

where $\sigma>0,0<k<\frac{1}{3}$, and $q$ is an arbitrary real number. Also, since $\sigma>0$, we can assume that $\varsigma \neq 0$.

Since the parameter $q$ in this section is assumed to be independent on $\varsigma$, the following results are similar to one-dimensional nonlinear elasticity problems studied by Gao [12, 13], Gao and Ogden [19].

Lemma 3.1 If $\varsigma_{1}, \varsigma_{2}, \varsigma_{3}$ are solutions of the equations $G\left(\varsigma, q, \sigma_{1}\right)=0, G\left(\varsigma, q, \sigma_{2}\right)=0$, $G\left(\varsigma, q, \sigma_{3}\right)=0$ correspondingly, and $\varsigma_{1}+\varsigma_{2}+\varsigma_{3}=q$, then $\varsigma_{1}, \varsigma_{2}, \varsigma_{3}$ satisfy $(46)$.

Proof. Obvious.

Lemma 3.2 Equation (47) has exactly one positive solution. It has negative solutions iff

$$
q \leq \frac{1}{k}\left(1-3 \sqrt[3]{\frac{\sigma}{4}}\right)
$$

There is only one negative solution if and only if $q=\frac{1}{k}\left(1-3 \sqrt[3]{\frac{\sigma}{4}}\right)$. 
Proof. To check that there is exactly one positive root one can apply the Descartes' rule of signs. To prove the rest, let's fix $q, \sigma$ and notice that $G(\varsigma, q, \sigma)=0$ has negative solutions iff it has at least two different solutions. This will happen iff the values of the function at local minimum and maximum have different signs. The extremums of $\mathrm{G}$ are at $\varsigma_{0}=-\frac{2}{3}(1-k q)$ and 0 . Since the value of $\mathrm{G}$ at 0 is $-\sigma<0$, we find when $G\left(\varsigma_{0}, q, \sigma\right) \geq 0$. Solving this inequality we get $q \leq \frac{1}{k}\left(1-3 \sqrt[3]{\frac{\sigma}{4}}\right)$.

Corollary 3.1 The equation $G(\varsigma, 0, \sigma)=0$ has negative solution(s) iff

$$
\sigma \leq \frac{4}{27}
$$

Proof. Apply Lemma 3.2 to $q=0$.

Lemma 3.3 Let's fix $\sigma>0$ and assume that $\varsigma_{0}, q_{0}$ satisfy $(47)$, and $\varsigma_{0} \neq 0, \varsigma_{0} \neq-\sqrt[3]{2 \sigma}$. Then there exists a unique continuously differentiable function $\varsigma(q)$, such that $\varsigma\left(q_{0}\right)=\varsigma_{0}$, $\varsigma(q)$ and $q$ both satisfy (47) and

$$
\frac{d \varsigma}{d q}=\frac{k \varsigma^{3}}{\varsigma^{3}+2 \sigma} .
$$

Moreover, there are three possibilities ("branches") for $\varsigma(q)$ :

(a) If $\varsigma_{0} \in(-\infty,-\sqrt[3]{2 \sigma})$, then the range of $\varsigma(q)$ is $(-\infty,-\sqrt[3]{2 \sigma})$, the domain is $\left(-\infty, \frac{1}{k}\left(1-3 \sqrt[3]{\frac{\sigma}{4}}\right)\right)$, and $\varsigma(q)$ is monotonically increasing.

(b) If $\varsigma_{0} \in(-\sqrt[3]{2 \sigma}, 0)$, then the range of $\varsigma(q)$ is $(-\sqrt[3]{2 \sigma}, 0)$, the domain is $\left.\left(-\infty, \frac{1}{k}\left(1-3 \sqrt[3]{\frac{\sigma}{4}}\right)\right)\right)$, and $\varsigma(q)$ is monotonically decreasing.

(c) If $\varsigma_{0} \in(0,+\infty)$, then the range of $\varsigma(q)$ is $(0,+\infty)$, the domain is $(-\infty,+\infty)$, and $\varsigma(q)$ is monotonically increasing.

Proof. Let's fix $\sigma$ and find $q$ from (47)

$$
q(\varsigma)=\frac{\varsigma^{3}+\varsigma^{2}-\sigma}{k \varsigma^{2}}
$$

Since, $\frac{d q}{d \varsigma}=\frac{\varsigma^{3}+2 \sigma}{k \varsigma^{3}}$ and $\sigma>0$ it is obvious that $q(\varsigma)$ is monotonically increasing in the intervals $\varsigma \in(-\infty,-\sqrt[3]{2 t})$ and $\varsigma \in(0,+\infty)$ and is monotonically decreasing in the interval 
$\varsigma \in(-\sqrt[3]{2 t}, 0)$. The corresponding intervals for $q$ are $\left(-\infty, \frac{1}{k}\left(1-3 \sqrt[3]{\frac{\sigma}{4}}\right)\right),(-\infty,+\infty)$, and $\left(-\infty, \frac{1}{k}\left(1-3 \sqrt[3]{\frac{\sigma}{4}}\right)\right)$. Also, one can easily check that

$$
\frac{d \varsigma}{d q}=\frac{k \varsigma^{3}}{\varsigma^{3}+2 \sigma}
$$

Thus, the lemma is proved.

Definition 3.1 The three branches of $\varsigma(q, \sigma)$ ( $\sigma$ is fixed) described in Lemma 3.3 will be denoted as follows:

(a) $\varsigma^{1}(q, \sigma)$ is a positive branch with the domain $(-\infty,+\infty)$ and range $(0,+\infty)$;

(b) $\varsigma^{3}(q, \sigma)<\varsigma^{2}(q, \sigma)$ are two negative branches with the domain $\left(-\infty, \frac{1}{k}\left(1-3 \sqrt[3]{\frac{\sigma}{4}}\right)\right)$ and ranges $(-\infty,-\sqrt[3]{2 \sigma})$ and $(-\sqrt[3]{2 \sigma}, 0)$ correspondingly. (Note that Corollary 3.1 implies that $\left.\frac{1}{k}\left(1-3 \sqrt[3]{\frac{\sigma}{4}}\right) \leq 0\right)$

Definition 3.2 Let's introduce the following notations:

(a) $\bar{\varsigma}^{i}(q, \sigma)=\varsigma^{i}(q, \sigma)-\frac{q}{3}, i=1,2,3$;

(b) $F^{i, j, k}\left(q, \sigma_{1}, \sigma_{2}, \sigma_{3}\right)=\bar{\varsigma}^{i}\left(q, \sigma_{1}\right)+\bar{\varsigma}^{j}\left(q, \sigma_{2}\right)+\bar{\varsigma}^{k}\left(q, \sigma_{3}\right), i, j, k=1,2,3$.

Lemma 3.4 The following statements are true:

(a) For $i=1,2,3$

$$
\bar{\varsigma}^{i}(q, \sigma)=-\frac{(1-3 k) \varsigma^{i}(q, \sigma)^{3}+\varsigma^{i}(q, \sigma)^{2}-\sigma}{3 k \varsigma^{i}(q, \sigma)^{2}}
$$

and

$$
\frac{d \bar{\varsigma}^{i}}{d q}=-\frac{(1-3 k) \varsigma^{i}(q, \sigma)^{3}+2 \sigma}{3\left(\varsigma^{i}(q, \sigma)^{3}+2 \sigma\right)} .
$$

(b) $\varsigma^{1}(0, \sigma)=\bar{\varsigma}^{1}(0, \sigma)>0, \varsigma^{2}(0, \sigma)=\bar{\varsigma}^{2}(0, \sigma)<0$, and $\varsigma^{3}(0, \sigma)=\bar{\varsigma}^{3}(0, \sigma)<0$.

(c) For a fixed $\sigma, \bar{\varsigma}^{-1}(q, \sigma)$ is monotonically decreasing in $q$ and $\lim _{q \rightarrow+\infty} \bar{\varsigma}^{-1}(q, \sigma)=-\infty$.

(d) For a fixed $\sigma$,

$$
\lim _{q \rightarrow-\infty} \bar{\varsigma}^{2}(q, \sigma)=+\infty \quad \text { and } \quad \lim _{q \rightarrow-\infty} \bar{\varsigma}^{3}(q, t)=+\infty
$$


(e) For fixed $\sigma_{1}, \sigma_{2}, \sigma_{3}$, each of $F^{i, j, k}\left(q, \sigma_{1}, \sigma_{2}, \sigma_{3}\right), i, j, k=1,2,3$, is continuous. Moreover, $F^{1,1,1}\left(q, \sigma_{1}, \sigma_{2}, \sigma_{3}\right)$ is monotonically decreasing in $q$.

Proof. To check (a), first substitute $q(\varsigma)=\frac{\varsigma^{3}+\varsigma^{2}-\sigma}{k \varsigma^{2}}$ into $\bar{\varsigma}^{i}(q, \sigma)=\varsigma^{i}(q, \sigma)-\frac{q}{3}, i=1,2,3$. Expression for $\frac{d \bar{\varsigma}^{i}}{d q}$ can be obtained either by direct differentiation of the previously obtained expression for $\varsigma^{i}(q, \sigma)$ or subtracting $\frac{1}{3}$ from $\frac{d \varsigma}{d q}=\frac{k \varsigma^{3}}{\varsigma^{3}+2 \sigma}$.

(b) is obvious.

To prove (c), recall, that $k<\frac{1}{3}$, and use formulas from (a). To prove (d), recall, that $k<\frac{1}{3}$, and use the first formula from (a). (e) immediately follows from (a) and (c).

Lemma 3.5 Solutions, $\varsigma^{1}(0, \sigma), \varsigma^{2}(0, \sigma), \varsigma^{3}(0, \sigma)$, of the equation $G(\varsigma, 0, \sigma)=\varsigma^{3}+\varsigma^{2}-\sigma=$ $0,0<\sigma \leq \frac{4}{27}$, enjoy the following properties:

(a) If $\sigma=\frac{4}{27}$ the solutions are $\varsigma^{1}\left(0, \frac{4}{27}\right)=\frac{1}{3}, \varsigma^{2}\left(0, \frac{4}{27}\right)=\varsigma^{3}\left(0, \frac{4}{27}\right)=-\frac{2}{3}$

(b) If $0<\sigma_{1}<\sigma_{2} \leq 0$, then

$$
0<\varsigma^{1}\left(0, \sigma_{1}\right)<\varsigma^{1}\left(0, \sigma_{2}\right) \leq \frac{1}{3}
$$

and

$$
-1<\varsigma^{3}\left(0, \sigma_{1}\right)<\varsigma^{3}\left(0, \sigma_{2}\right) \leq-\frac{2}{3} \leq \varsigma^{2}\left(0, \sigma_{2}\right)<\varsigma^{2}\left(0, \sigma_{1}\right)<0
$$

(c) $\varsigma^{1}(0, \sigma)+\varsigma^{2}(0, \sigma)<0$

Proof. (a) can be checked directly.

To prove (b), one can either apply the implicit function theorem to $H(\varsigma, \sigma)=G(\varsigma, 0, \sigma)=0$. Or, less formally, draw the graph of $y=\varsigma^{3}+\varsigma^{2}-\frac{4}{27}$ and observe what happens to its roots when the graph is shifted upward until it becomes $y=\varsigma^{3}+\varsigma^{2}$.

(c) Obviously, $\varsigma^{1}(0, \sigma)+\varsigma^{2}(0, \sigma)+\varsigma^{3}(0, \sigma)=-1$. So, $\varsigma^{1}(0, \sigma)+\varsigma^{2}(0, \sigma)=-1-\varsigma^{3}(0, \sigma)<0$, since $\varsigma^{3}(0, \sigma)>-1$.

\subsection{Solutions of the St. Venant-Kirchhoff Material}

We are now ready to present our main results.

Theorem 3.1 For any given force field $\mathbf{f}: \Omega \rightarrow \mathbb{R}^{d}$ and the surface traction $\mathbf{t}: \Gamma_{t} \rightarrow \mathbb{R}^{d}$ such that the statically admissible stress $\boldsymbol{\tau} \in \mathcal{T}_{a}$ has no zero eigenvalues almost ever where in $\Omega$, the canonical dual problem $\left(\mathcal{P}^{d}\right)$ has a unique positive critical solution $\mathbf{S} \in \mathcal{S}_{a}^{+}$. 
Proof. We need to prove that for arbitrarily given $\sigma_{1}, \sigma_{2}, \sigma_{3}>0$, the system of equations (46) has a unique positive solution $\left(\varsigma_{1}, \varsigma_{2}, \varsigma_{3}\right)$, such that all $\varsigma_{i}>0, i=1,2,3$. From Lemma $3.4(\mathrm{~b})$, it follows that

$$
F^{1,1,1}\left(0, \sigma_{1}, \sigma_{2}, \sigma_{3}\right)>0 .
$$

From Lemma 3.4(c), it follows that for some $q_{1}>0$, large enough,

$$
F^{1,1,1}\left(q_{1}, \sigma_{1}, \sigma_{2}, \sigma_{3}\right)<0
$$

Therefore, since $F^{1,1,1}$ is continuous and monotonically decreasing in $q$ (Lemma 3.4(e)), there exists a unique $q_{0}, 0<q_{0}<q_{1}$, such that

$$
F^{1,1,1}\left(q_{0}, \sigma_{1}, \sigma_{2}, \sigma_{3}\right)=0 .
$$

i.e.

$$
\varsigma^{1}\left(q_{0}, \sigma_{1}\right)+\varsigma^{1}\left(q_{0}, \sigma_{2}\right)+\varsigma^{1}\left(q_{0}, \sigma_{3}\right)=q_{0} .
$$

So, from Lemma 3.1 it follows that $\varsigma^{1}\left(q_{0}, \sigma_{1}\right), \varsigma^{1}\left(q_{0}, \sigma_{2}\right), \varsigma^{1}\left(q_{0}, \sigma_{3}\right)$ form a positive solution of (46), which are eigenvalues of the second Piola-Kirchhoff stress S. Therefore, Problem $\left(\mathcal{P}^{d}\right)$ has a unique global maximizer $\mathbf{S} \in \mathcal{S}_{a}^{+}$.

Theorem 3.2 For any given force field $\mathbf{f}: \Omega \rightarrow \mathbb{R}^{d}$ and the surface traction $\mathbf{t}: \Gamma_{t} \rightarrow \mathbb{R}^{d}$ such that the eigenvalues of the statically admissible stress tensor function $\boldsymbol{\tau} \in \mathcal{T}_{a}$ satisfy $0<\sigma_{1}, \sigma_{2}, \sigma_{3}<\frac{4}{27}$, the total complementary energy $\Pi_{\boldsymbol{\tau}}^{d}(\mathbf{S})$ has eight negative solutions $\mathbf{S}_{k} \in \mathcal{S}_{a}^{-}, k=1, \ldots, 8$.

Proof. We need to prove that for arbitrarily given $0<\sigma_{1}, \sigma_{2}, \sigma_{3}<\frac{4}{27}$, the system of equations (46) has 8 solutions $\left(\varsigma_{1}, \varsigma_{2}, \varsigma_{3}\right)$, such that all $\varsigma_{i}<0, i=1,2,3$. From Corollary 3.1 it follows that each of the equations $G\left(\varsigma, 0, \sigma_{i}\right)$, has two negative solutions, $\varsigma^{2}\left(0, \sigma_{i}\right)>$ $\varsigma^{3}\left(0, \sigma_{i}\right), i=1,2,3$. From Lemma 3.4(b), it follows that for $i, j, k=2,3$

$$
F^{i, j, k}\left(0, \sigma_{1}, \sigma_{2}, \sigma_{3}\right)<0 .
$$

From Lemma 3.4(d) it follows that there exists $q_{1}<0$ such that

$$
F^{i, j, k}\left(q_{1}, \sigma_{1}, \sigma_{2}, \sigma_{3}\right)>0 .
$$

Therefore, since $F^{i, j, k}$ is continuous in $q$ (Lemma $3.4(\mathrm{e})$ ), there exists $q_{0}, 0>q_{0}>q_{1}$, such that

$$
F^{i, j, k}\left(q_{0}, \sigma_{1}, \sigma_{2}, \sigma_{3}\right)=0
$$


i.e.

$$
\varsigma^{i}\left(q_{0}, \sigma_{1}\right)+\varsigma^{j}\left(q_{0}, \sigma_{2}\right)+\varsigma^{k}\left(q_{0}, \sigma_{3}\right)=q_{0} .
$$

So, from Lemma 3.1 it follows that $\varsigma^{i}\left(q_{0}, \sigma_{1}\right), \varsigma^{j}\left(q_{0}, \sigma_{2}\right), \varsigma^{k}\left(q_{0}, \sigma_{3}\right)$ form a negative solution of (46). Since, each of $i, j, k$ can be chosen independently from the set $\{2,3\}$, we have total 8 different negative solutions.

Theorem 3.3 For any given force field $\mathbf{f}: \Omega \rightarrow \mathbb{R}^{d}$ and the surface traction $\mathbf{t}: \Gamma_{t} \rightarrow \mathbb{R}^{d}$ such that the eigenvalues of the statically admissible stress tensor function $\boldsymbol{\tau} \in \mathcal{T}_{\text {a }}$ satisfy $0<\sigma_{1}, \sigma_{2}, \sigma_{3}<\frac{4}{27}$, the total complementary energy $\Pi_{\boldsymbol{\tau}}^{d}(\mathbf{S})$ has at least 15 mixed stationary points, i.e., some eigenvalues $\varsigma_{i}, i=1,2,3$, of $\mathbf{S}$ are positive, some are negative.

Proof. Each of the equations $G\left(\varsigma, 0, \sigma_{i}\right)$, has one positive and two negative solutions: $\varsigma^{1}, \varsigma^{2}, \varsigma^{3}$. Applying Lemma 3.5 it is easy to check that

(1) for $i, j=2,3$,

$$
F^{1, i, j}\left(0, \sigma_{1}, \sigma_{2}, \sigma_{3}\right)<0, F^{i, 1, j}\left(0, \sigma_{1}, \sigma_{2}, \sigma_{3}\right)<0
$$

(2) $F^{2,3,1}\left(0, \sigma_{1}, \sigma_{2}, \sigma_{3}\right)<0, F^{3,2,1}\left(0, \sigma_{1}, \sigma_{2}, \sigma_{3}\right)<0, F^{3,3,1}\left(0, \sigma_{1}, \sigma_{2}, \sigma_{3}\right)<0$.

(3) $F^{1,1,2}\left(0, \sigma_{1}, \sigma_{2}, \sigma_{3}\right)<0, F^{1,1,3}\left(0, \sigma_{1}, \sigma_{2}, \sigma_{3}\right)<0, F^{1,3,1}\left(0, \sigma_{1}, \sigma_{2}, \sigma_{3}\right)<0$, $F^{3,1,1}\left(0, \sigma_{1}, \sigma_{2}, \sigma_{3}\right)<0$.

For each of these 15 combinations, $F^{a, b, c}$, there exists $q_{1}<0$ such that $F^{a, b, c}\left(q_{1}, \sigma_{1}, \sigma_{2}, \sigma_{3}\right)>$ 0 .

Therefore, since $F^{a, b, c}$ is continuous in $q$ (Lemma 3.4(e)), there exists $q_{0}, 0>q_{0}>q_{1}$, such that

$$
F^{a, b, c}\left(q_{0}, l_{1}, l_{2}, l_{3}\right)=0
$$

that is

$$
\varsigma^{a}\left(q_{0}, \sigma_{1}\right)+\varsigma^{b}\left(q_{0}, \sigma_{2}\right)+\varsigma^{c}\left(q_{0}, \sigma_{3}\right)=q_{0}
$$

So, from Lemma 3.1 it follows that $\varsigma^{a}\left(q_{0}, \sigma_{1}\right), \varsigma^{b}\left(q_{0}, \sigma_{2}\right), \varsigma^{c}\left(q_{0}, \sigma_{3}\right)$ form a mixed solution of (46).

Obviously, these 15 combinations result in different mixed stationary points of $\Pi_{\boldsymbol{\tau}}^{d}(\mathbf{S})$. 


\section{Conclusions}

We have illustrated that by using the canonical duality theory, the nonconvex minimal potential problem $(\mathcal{P})$ is canonically dual to a concave maximization problem in a convex

stress space $\mathcal{S}_{a}^{+}$, which can be solved by well-developed numerical methods. By the pure complementary energy principle, the general nonlinear partial differential equation in nonlinear elasticity is actually equivalent to an algebraic (tensor) equation, which can be solved for certain materials to obtain all possible stress solutions. Both global and local extremal solutions can be identified by the triality theory, while the Legendre-Hadamard condition is only necessary for local minimizers. Our results shows that for St. Venant-Kirchhoff material, the nonlinear boundary value problem could have 24 solutions at each material point, but only one global minimizer if the statically admissible stress $\tau \neq 0$. It is important to have a detailed study on these solutions in the future.

\section{Acknowledgements}

The research of the first author was supported by the US Air Force Office of Scientific Research under the grant AFOSR FA9550-10-1-0487. Results presented in Section 3 were discussed with Professor Ray Ogden from University of Glasgow.

\section{References}

[1] Abeyaratne, R. C. 1980 Discontinuous deformation gradients in plane finite elastostatics of incompressible materials. J. Elasticity 10, 255-293.

[2] Abeyaratne, R. C. 1981 Discontinuous deformation gradients in the finite twisting of an incompressible elastic tube. J. Elasticity 11, 43-80.

[3] Arthurs, A.M. (1980). Complementary Variational Principles, Clarendon Press, Oxford.

[4] Cai, K., Gao, DY, Qin, QH (2014). Post-buckling solutions of hyper-elastic beam by canonical dual finite element method, Mathematics and Mechanics of Solids. 19(6) 659-671

[5] Ciarlet, P.G. (1988). Mathematical Elasticity. Volume I: Three-Dimensional Elasticity. North-Holland. 
[6] Gao, D. Y. 1992 Global extremum criteria for nonlinear elasticity. J. Appl. Math. Physics ( ZAMP) 43, 924-937.

[7] Gao, D. Y. 1997 Dual extremum principles in finite deformation theory with applications to post-buckling analysis of extended nonlinear beam theory. Appl. Mech. Rev. 50, S64-S71.

[8] Gao, D. Y. 1998 Duality, triality and complementary extremum principles in nonconvex parametric variational problems with applications. IMAJ. Appl. Math. 61, 199-235.

[9] Gao, D. Y. 1999a Pure complementary energy principle and triality theory in finite elasticity. Mech. Res. Comm. 26, 31-37.

[10] Gao, D. Y. 1999b Duality-Mathematics. Wiley Encyclopedia of Electronical and Electronical Engineering 6, 68-77.

[11] Gao, D. Y. 1999c General analytic solutions and complementary variational principles for large deformation nonsmooth mechanics. Meccanica 34, 169-198.

[12] Gao, D. Y. 2000a Duality Principles in Nonconvex Systems: Theory, Methods and Applications. Kluwer Academic Publishers, Dordrecht /Boston /London, xviii + 454pp.

[13] Gao, D. Y. 2000b Analytic solution and triality theory for nonconvex and nonsmooth variational problems with applications. Nonlinear Analysis 42, 1161-1193.

[14] Gao, D. Y. 2000c Canonical dual transformation method and generalized triality theory in nonsmooth global optimization J. Global Optimization 17, 127-160.

[15] Gao, D. Y. 2003a Nonconvex semi-linear problems and canonical dual solutions. Advances in Mechanics and Mathematics, Vol. II, D. Y. Gao \& R. W. Ogden (eds), Kluwer Academic Publishers, pp. 261-312.

[16] Gao, D. Y. 2003b Perfect duality theory and complete set of solutions to a class of global optimization. Optimization 52, 467-493.

[17] Gao, D. Y. 2004 Complementary variational principle, algorithm, and complete solutions to phase transitions in solids governed by Landau-Ginzburg equation. Math. Mech. Solids 9, 285-305. 
[18] Gao, D.Y. 2009 Canonical duality theory: unified understanding and generalized solutions for global optimization. Comput. \& Chem. Eng. 33, 1964-1972.

[19] Gao, D. Y. \& Ogden, R. W. 2008. Multiple solutions to non-convex variational problems with implications for phase transitions and numerical computation. Q. J. Mech. Appl. Math. 61, 497-522.

[20] Gao, D.Y. and Ogden, R.W. 2008. Closed-form solutions, extremality and nonsmoothness criteria in a large deformation elasticity problem, ZAMP, 59:498 - 517.

[21] Gao, D. Y. \& Ruan, N. 2010 Solutions to quadratic minimization problems with box and integer constraints. J. Global Optim. 47, 463-484.

[22] Gao, D. Y., Ruan, N. and Latorre, V. 2015. Canonical duality-triality: Bridge between nonconvex analysis/mechanics and global optimization, Math. Mech. Solids.

[23] Gao, D. Y. \& Strang, G. 1989 Geometric nonlinearity: potential energy, complementary energy, and the gap function. Q. Appl. Math. 47, 487-504.

[24] Gao, D.Y., Yu, H.F. (2008). Multi-scale modelling and canonical dual finite element method in phase transitions of solids. Int. J. Solids Struct. 45, 3660-3673.

[25] Hellinger, E. 1914 Die allgemeine Ansätze der Mechanik der Kontinua. Encyklopädie der Mathematischen Wissenschaften IV, 4, 602-694.

[26] Jiang, X. \& Ogden, R. W. 1998 On azimuthal shear of a circular cylindrical tube of compressible elastic material. Q. J. Mech. Appl. Math. 51, 143-158.

[27] Kassianidis, F., Merodio, J., Ogden, R. W. \& Pence, T. J. 2008 Azimuthal shear of a transversely isotropic elastic solid. Math. Mech. Solids 13, 690-724.

[28] Koiter, W. T. 1976 On the complementary energy theorem in nonlinear elasticity theory. In Trends in Appl. of Pure Math. to Mech., G. Fichera (ed.), Pitman.

[29] Lee, S. J. \& Shield, R. T. 1980a Variational principles in finite elastics. J. Appl. Math. Physics (ZAMP) 31, 437-453.

[30] Lee, S. J. \& Shield, R. T. 1980 Applications of variational principles in finite elasticity. J. Appl. Math. Physics (ZAMP) 31, 454-472. 
[31] Levinson, M. 1965 The complementary energy theorem in finite elasticity. J. Appl. Mech. 87, 826-828.

[32] Li, S. F. \& Gupta, A. 2006 On dual configuration forces. J. Elasticity 84, 13-31.

[33] Li, C., Zhou, X., and Gao DY (2014). Stable trajectory of logistic map, Nonlinear Dynamics, DOI 10.1007/s11071-014-1433-y

[34] Noble, B. and Sewell, M.J. (1972). On dual extremum principles in applied mathematics, J. Inst. Math. Appl., 9, 123-193.

[35] Oden, J. T. \& Reddy, J. N. 1983 Variational Methods in Theoretical Mechanics. Springer-Verlag.

[36] Ogden, R. W. 1975 A note on variational theorems in non-linear elastostatics. Math. Proc. Camb. Phil. Soc. 77, 609-615.

[37] Ogden, R. W. 1977 Inequalities associated with the inversion of elastic stressdeformation relations and their implications. Math. Proc. Camb. Phil. Soc. 81, 313-324.

[38] Reissner, E. 1953 On a variational theorem for finite elastic deformations. J. Math. Phys. 32, 129-135.

[39] Rivlin, R. S. 1949 Large elastic deformations of isotropic materials VI. Further results in the theory of torsion, shear and flexure. Phil. Trans. R. Soc. Lond. A 242, 173-195.

[40] Ruan, N. and Gao, D.Y. (2014). Global optimal solutions to a general sensor network localization problem, Performence Evaluations, 75-76: 1-16.

[41] Ruan, N. and Gao, D.Y.(2014). Canonical duality approach for nonlinear dynamical systems, IMA J. Appl. Math., 79: 313-325.

[42] Ruan, N., Gao, D. Y. \& Jiao, Y. 2010 Canonical dual least square method for solving general nonlinear systems of equations. Comput. Optim. Appl. 47, 335-347.

[43] Santos, H.A.F.A. and Gao D.Y. (2011). Canonical dual finite element method for solving post-buckling problems of a large deformation elastic beam, Int. J. Nonlinear Mechanics, 47: 240 - 247. doi:10.1016/j.ijnonlinmec.2011.05.012 
[44] Tabarrok, B. and Rimrott, F.P. (1994). Variational Methods and Complementary Formulations in Dynamics, Springer, 366pp.

[45] Truesdell C. and Noll W.: The Non-Linear Field Theories of Mechanics. Springer Verlag, third edition, 2004.

[46] Veubeke, B. F. 1972 A new variational principle for finite elastic displacements. Int. J. Eng. Sci. 10, 745-763.

[47] Zhang J., Gao, D.Y. and Yearwood, J. (2011). A novel canonical dual computational approach for prion AGAAAAGA amyloid fibril molecular modeling. Journal of Theoretical Biology, 284, 149-157 (2011). doi:10.1016/j.jtbi.2011.06.024 